\title{
0190 LINKING TRAUMA REGISTRY INJURY DATA WITH NATIONAL INJURY COMPENSATION DATA - OPPORTUNITIES FOR PUBLIC HEALTH ACTION IN NEW ZEALAND
}

B Kool, ${ }^{*}$ C Jackson, I Civil, R Dansey, S Ameratunga Correspondence: Section Epidemiology \& Biostats, School of Population Health, University of Auckland, Auckland, New Zealand

10.1136/ip.2010.029215.190

Background Trauma registries can play a key role in quality assurance and planning of trauma care. This study aimed to explore the feasibility and utility of linking data from a trauma registry to the national injury compensation scheme to estimate the impact of major trauma beyond the hospitalisation.

Methods Using a deterministic linkage approach that included the unique national master patient index number, trauma registry data from adult patients admitted for acute care to Auckland City Hospital over a 22 month period (1 April 2006 to 31 January 2008) was linked to Accident Compensation Corporation data. Descriptive analysis investigated the quality, 
potential biases and the utility of this approach to achieve the study aims.

Results A successful link was achieved for $72.4 \%$ ( $n=1520 / 2099$ ) of trauma patients meeting the study criteria. Factors affecting the success of linkage included: ethnicity, TRISS scores, admission duration, and survival to discharge. Eighty-three percent of patients with linked data had returned to work within 12 months of injury. Those most likely to receive compensated time off work included machinery operators/drivers, and those injured due to land transport accidents. Cases least likely to have returned to work at 3 months were labourers, those injured in land transport incidents, and cases with ISS $>15$.

Conclusions High quality, robust data linkage that, in the future, also includes probabilistic approaches, have the potential to inform priority setting in injury prevention, and aid in evaluating the quality and effectiveness of acute trauma care and rehabilitation services. 